\title{
Metastatic basal cell carcinoma: A report of two cases and a review of the literature
}

\section{Claire Quigley, Siona Ni Raghallaigh}

\author{
Departments of Dermatology, Beaumont Hospital, Dublin, Ireland
}

Corresponding author: Claire Quigley, MD, E-mail: quiglec3@tcd.ie

\begin{abstract}
Basal cell carcinomas (BCCs) are among the most common non-melanoma skin cancers in the world. However, given their slowly progressive nature, metastatic BCCs are a relatively uncommon entity. Below, we discuss two separate cases of metastatic BCC that we encountered in our clinical practice. The first is the case of a 57-year-old male with a right cheek BCC and bilateral pulmonary metastases. The second is the case of a 71 -year-old male who also presented with a right $\mathrm{BCC}$ and pulmonary metastases. We discuss their altered clinical courses. We also conducted a review of the literature focusing on the use of the relatively novel hedgehog inhibitors as a treatment option for individuals diagnosed with metastatic BCC.
\end{abstract}

Key words: BCC; metastatic; hedgehog inhibitors

\section{INTRODUCTION}

It is estimated that approx. $50 \%$ of referrals to the dermatologist are for skin cancer [1]. Basal cell carcinoma (BCC) is the most common cancer worldwide and accounts for $80 \%$ of non-melanoma skin cancers. An increasing age and prolonged sun exposure are significant risk factors for the development of BCC.

Herein, we present two cases of metastatic BCC, a rare progression of a common presentation. We also review the literature to identify what features could be used to stratify patients into high-risk and low-risk according to the progression of metastatic BCC and what treatment options are available to those with this diagnosis today.

\section{CASE REPORTS}

\section{Case 1}

The first is a case of a 57-year-old male who presented with a $2 \times 2 \mathrm{~cm}$ lesion on the right cheek clinically consistent with BCC. He had a palpable lymph node in his right cervical chain and had a history of atrial fibrillation, hypertension, coronary artery disease, insulin-dependent diabetes mellitus, and diabetic nephropathy, as well as a previous basal cell carcinoma removal from the left nasal ala one year before presentation. The patient was a heavy smoker with a 30-pack-year history.

CT imaging of the neck revealed a $2.6 \mathrm{~cm}$ exophytic soft tissue lesion on the right cheek, which was in keeping with the known primary $\mathrm{BCC}$, as well as a $3 \mathrm{~cm}$ mass in the right neck, concerning local invasion. A brain, abdomen, and pelvis CT scan showed no evidence of distal metastases.

Under the care of a plastic surgeon, the patient underwent excision of the BCC from the right cheek (Fig. 1) and FNA of LN followed by a neck dissection of levels I, II, and III with superficial parotidectomy. Histology (Fig. 2) showed mixed nodular and infiltrative subtypes of BCC. Three of the twenty-five lymph nodes were positive for metastatic carcinoma and one node was positive at level II at the tail of the parotid gland.

Although no systemic therapy was recommended, the patient completed a course of RT with 60GY over thirty fractions.

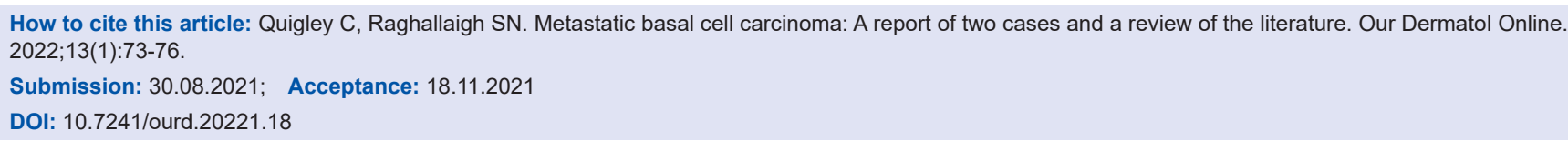


Around one year later, the patient presented again with a localized recurrence of BCC on the right cheek and palpable right anterior cervical lymph nodes. A biopsy confirmed dermal foci of BCC in the right cheek. CT of the head and neck showed an infiltrative mass in the right cheek and a second, more solid and discrete mass in the right preauricular soft tissue adjacent to the right masseter muscle, suggestive of a local recurrence of the tumor or localized metastatic lymphadenopathy. Abnormal right paratracheal lymph nodes and a large right pleural effusion were also noted. There was no evidence of brain disease. CT of the abdomen and pelvis showed evidence of widespread metastatic disease with a large right pleural effusion and multiple pulmonary and pleural nodes and masses, mediastinal and right hilar lymphadenopathy, hepatic metastases, and subcutaneous enhancing nodules.

Analysis of the pleural effusion revealed a poorly cellular fluid showing single and small groups of mesothelial cells with reactive changes. Mixed-background inflammatory cells, including macrophages, were present. No malignant cells were seen. An incisional biopsy of the left flank nodule (Fig. 3) was consistent with $\mathrm{BCC}$, confirming metastatic BCC.

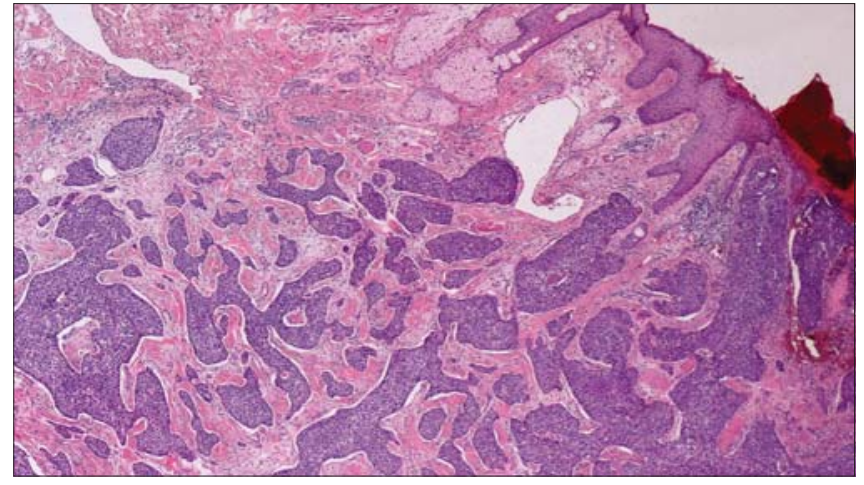

Figure 1: Large basal cell carcinoma, the mixed nodular and infiltrative types, from the cheek measuring $13 \mathrm{~mm}$ in depth and extending to the deep aspect of the subcutis (H\&E; 2x; objective lens).

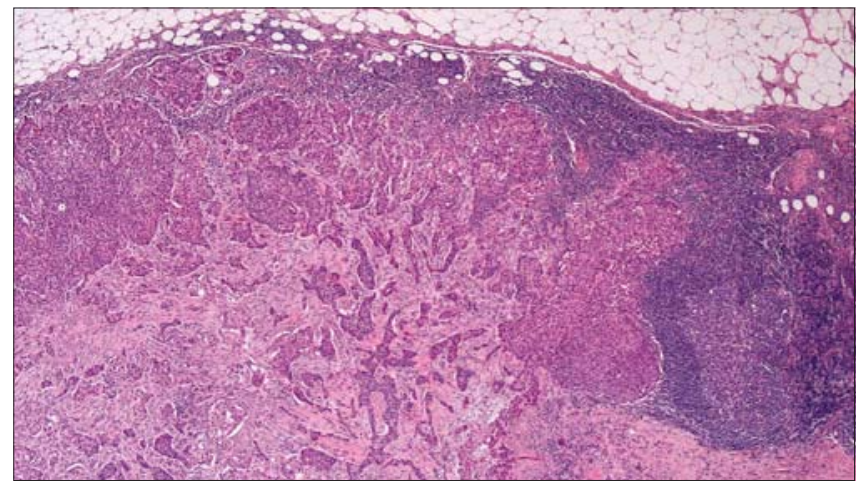

Figure 2: At the time of surgery, the neck dissection showed metastatic basal cell carcinoma in three lymph nodes (levels 1-3) (H\&E; 2x; objective lens).
Given the patient's multimorbid background history and the extent of his metastatic disease, he was not a candidate for any further surgery. He deteriorated clinically while in an inpatient in the hospital and died soon after admission.

\section{Case 2}

A 71-year-old male was referred to our tertiary center with a biopsy proving a $15 \mathrm{~cm}$ BCC on the right cheek present for twelve years. This was extending into the temporal bone with associated right-sided facial nerve palsy. His entire lateral skull was eroded, the distal auditory canal was visible, and the middle cranial fossa was exposed. There was a large palpable lymph node on the right side of the neck.

He was a smoker with a 20-pack-year history and had a history of alcohol abuse (Fig. 4).

The patient began pre-operative work-up for an urgent excision with flap and neck dissection. As part of the pre-op work-up, the patient had a chest X-ray, which revealed a $2.7 \mathrm{~cm}$ right-middle lobe lesion. TAP and PET CT confirmed the lung lesion and there was an avid FDG uptake in the middle lobe. Following FNS, a lobectomy confirmed metastatic BCC (Fig. 5).

In February 2020, the patient had a repeat PET and MRI and his imaging was discussed at an MDT, the outcome of which was that this patient's primary $\mathrm{BCC}$ was unresectable and best treated with chemotherapy.

He was referred to oncology and initiated on vismodegib in October 2020. He is currently on vismodegib and doing well. His primary BCC has improved since commencing vismodegib and he now requires fewer dressing changes for the lesion.

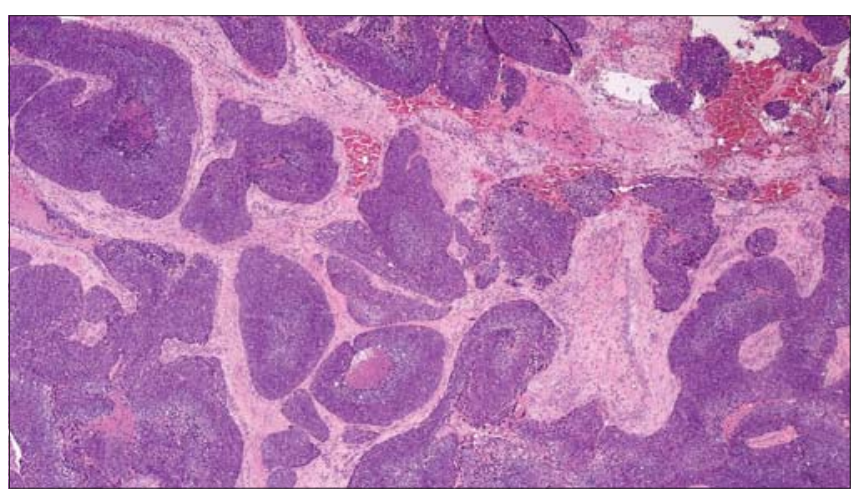

Figure 3: Incisional biopsy from the flank one year later showed a subcutaneous deposit of basal cell carcinoma (H\&E; 2x; objective lens). 
A recent restaging CT showed a new nodularity in the right lung base, which was slightly irregular and felt to be infectious or inflammatory, but interval imaging of this was recommended. There was no CT evidence of metastatic disease in the abdomen or pelvis.

\section{DISCUSSION}

Basal cell carcinoma (BCC) is the most common malignancy worldwide and accounts for $80 \%$ of all nonmelanoma skin cancers. However, despite its prevalence, metastases are exceedingly rare, having an incident rate between $0.0028 \%$ and $0.55 \%$. First described by Beadles in 1894 [1], metastatic BCC has a poor prognosis. For patients with locoregional lymphatic metastases, the mean survival is three years, this falls to only eight

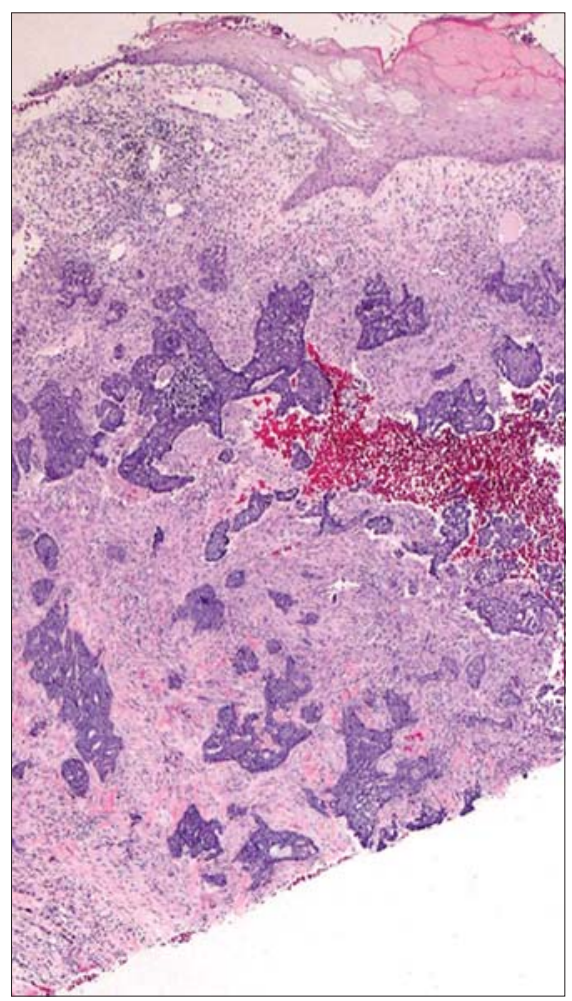

Figure 4: A biopsy from the right temple showing mixed nodular and infiltrative type basal cell carcinoma (H\&E; 2x; objective lens).

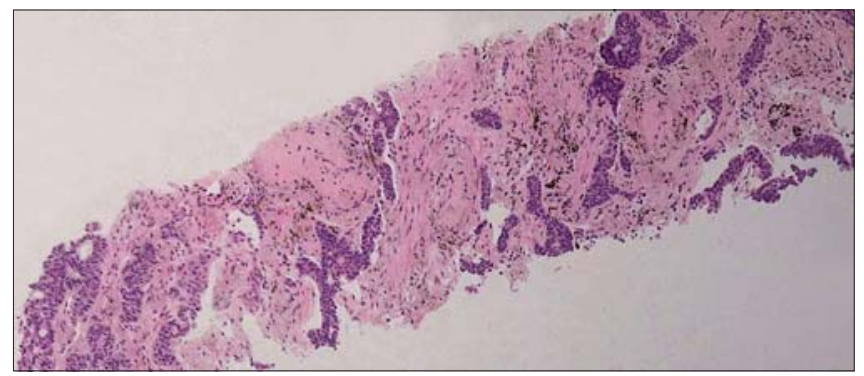

Figure 5: Core biopsy from the right upper lobe of the lung showing metastatic basal cell carcinoma (H\&E; 4x; objective lens). months for patients with distant metastatic disease. BCCs most frequently occur on areas of the skin that have experienced prolonged sun exposure, most commonly the face, neck, and hands. Large $(>10 \mathrm{~cm})$ [2] primary tumors, the invasion of the blood vessels or of the perineural spaces, a location in the head and neck region, multiple recurring or primary tumors, the condition after radiotherapy, immunosuppression, and fair skin as well as the male sex have been described as risk factors of developing a metastatic BCC [1]. It is worth to note that both our patients were fair-skinned males over fifty with large primary tumors in the head and neck region.

Metastatic BCC most commonly spreads to local lymph nodes $(60 \%)$, yet hematogenous spread to the lungs, bones, and other cutaneous sites has also been reported. The lungs are the most often involved, with almost fifty cases reported to date [3]. Interestingly, however, most cases involving the lungs are multiple small, disseminated nodules as the spread is hematogenous, in the case of our patients. Patient A presented with pleural nodules and a right upper bronchus lesion and patient $\mathrm{B}$ presented with an isolated right middle lobe lesion. Given their associated smoking history, both were initially considered to have a possible synchronous primary lung malignancy. As outlined above, both patients were subsequently diagnosed with biopsy-proven metastatic BCC.

Dandurand et al. (Table 1) provided a clinical practice guideline to enable clinicians to identify patients considered higher-risk. Consideration needs to be given to tumor location, size, and histology, whether or not this is a recurrent $\mathrm{BCC}$, in order to stratify it by risk.

For all patients with a BCC excised, a single follow-up appointment 6-12 months afterward is recommended. For patients who fall into the high-risk category, a

Table 1: From Dandurand et al 2006 [7].

\begin{tabular}{|c|c|c|}
\hline Low-risk BCC & Intermediate-risk BCC & High-risk BCC \\
\hline $\begin{array}{l}\text { Superficial primary } \\
\text { BCC }\end{array}$ & Superficial recurrent BCC & $\begin{array}{l}\text { Morpheaform or poor- } \\
\text { defined } B C C\end{array}$ \\
\hline $\begin{array}{l}\text { Nodule primary } \\
\mathrm{BCC} \text { when: } \\
<1 \mathrm{~cm} \text { in an } \\
\text { intermediate-risk } \\
\text { area } \\
<2 \mathrm{~cm} \text { in a low- } \\
\text { risk area }\end{array}$ & $\begin{array}{l}\text { Nodular primary } \mathrm{BCC} \text { when: } \\
<1 \mathrm{~cm} \text { in a high-risk area } \\
>1 \mathrm{~cm} \text { in an intermediate- } \\
\text { risk area } \\
>2 \mathrm{~cm} \text { in a low-risk area }\end{array}$ & $\begin{array}{l}\text { Nodular primary } \mathrm{BCC} \\
\text { when: } \\
>1 \mathrm{~cm} \text { in a high-risk } \\
\text { area }\end{array}$ \\
\hline Pink tumor & & $\begin{array}{l}\text { Histological forms: } \\
\text { aggressive } \\
\text { recurrent forms (apart } \\
\text { from superficial BCC) }\end{array}$ \\
\hline
\end{tabular}

High-risk zones are the nose and the periorificial areas of the head and neck. Intermediate-risk zones are the forehead, cheek, chin, scalp, and neck. Low-risk zones are the trunk and limbs. Aggressive histological forms include micronodular, morpheaform, and metatypical basosquamous forms. Perineural invasion also seems to be a histological sign of aggressiveness 


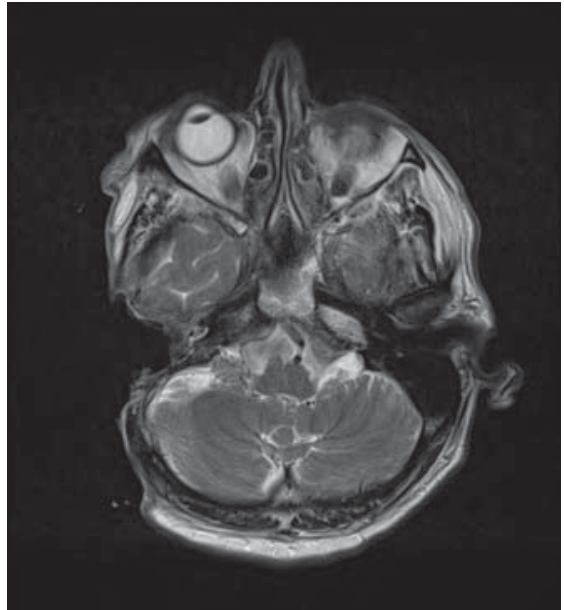

Figure 6: 1) Pre-operative CT imaging showing an extensive erosion of the right mastoid temporal bone and the middle ear cavity extending anteriorly to the right temporomandibular joint and zygomatic arch and posteriorly to the right lateral wall of the posterior fossa. 2) Dural involvement with a pachymeningeal thickening and enhancement overlying the right temporal lobe and right cerebellum. 3) Rim enhancing, thick-walled right cervical level-two mass, most likely a nodal metastasis.

follow-up every 6-12 months for 3-5 years is advised and annually thereafter [4].

Treatment options for BCC vary depending on a number of factors, including location, morphology, risk stratification, and patient factors, such as a multimorbid burden. For patients with metastatic disease, resection of the primary tumor, if possible, is advised. However, in recent years, new drugs have revolutionized the treatment of this rare entity.

Sporadic BCC is characterized by key genetic defects in the Hedgehog $(\mathrm{Hh})$ pathway, including loss of function mutations in PTCHl (9q22.3) in approx. 90\% of BCC tumors and activating mutations in the G-protein coupled receptor smoothened (SMO) in 10\% of BCCs [5]. The manipulation of this pathway, and the development of Hedgehog pathway inhibitors (HPIs) play a crucial role in the treatment of metastatic BCC, both for locoregional metastases and distant metastases. Vismodegib and sonidegib are both oral medications that act as HPIs. Vismodegib is currently licensed for the treatment of both distant metastatic disease and locally advanced disease. Sonidegib is currently only licensed for locally advanced disease. There are limited reports on their efficacy. Dessiniotti et al. reported a response rate of 48.5\% [5] to vismodegib for patients with metastatic BCC. The ERIVANCE study looked at the efficacy and safety of vismodegib for patients with locally advanced BCC (BCC with metastatic spread to regional lymph nodes) and metastatic BCC (BCC with distant metastases). The estimated median PFS by IRF assessment was 9.5 months for metastatic BCC [6]. The median overall survival for patients taking vismodegib with metastatic BCC was 24 months. This is an improvement in the predicted eight-month survival without HPIs.

Muscle spasms, dysgeusia, alopecia, nausea, and weight loss have been the most common side effects reported to date. Adverse side effects were most commonly seen within the first six months and less likely thereafter. Routine follow-up with oncology is required for all patients on systemic therapy as well as interval imaging.

\section{Consent}

The examination of the patient was conducted according to the principles of the Declaration of Helsinki.

The authors certify that they have obtained all appropriate patient consent forms, in which the patients gave their consent for images and other clinical information to be included in the journal. The patients understand that their names and initials will not be published and due effort will be made to conceal their identity, but that anonymity cannot be guaranteed.

\section{REFERENCES}

1. Wahl RU, Cacchi C, Rübben A. Cutaneous basal cell carcinoma with lymph node and pulmonary metastases. Case Rep Oncol Med. 2018;2018:3485326.

2. Moser S, Borm J, Mihic-Probst D, Jacobsen C, Kruse Gujer AL. Metastatic basal cell carcinoma: Report of a case and review of the literature. Oral Surg Oral Med Oral Pathol Oral Radiol. 2014;117:e79-82.

3. Seo SH, Shim WH, Shin DH, Kim YS, Sung HW. Pulmonary metastasis of basal cell carcinoma. Ann Dermatol. 2011;23:213-6.

4. Peris K, Fargnoli MC, Garbe C, Kaufmann R, Bastholt L, Seguin NB, et al. Diagnosis and treatment of basal cell carcinoma: European consensus-based interdisciplinary guidelines. Eur J Cancer. 2019;118:10-34.

5. Dessinioti C, Plaka M, Soura E, Mortaki D, Papaxoinis G, Gogas H, et al. A practical guide for the follow-up of patients with advanced basal cell carcinoma during treatment with Hedgehog pathway inhibitors. Oncologist. 2019;24:e755-e64.

6. Sekulic A, Migden MR, Lewis K, Hainsworth JD, Solomon JA, Yoo S, et al. Pivotal ERIVANCE basal cell carcinoma (BCC) study: 12-month update of efficacy and safety of vismodegib in advanced BCC. J Am Acad Dermatol. 2015;72:1021-6.e8.

7. Dandurand M, Petit T, Martel P, Guillot B, ANAES. Management of basal cell carcinoma in adults: Clinical practice guidelines. Eur J Dermatol. 2006;16:394-401.

Copyright by Claire Quigley, et al. This is an open-access article distributed under the terms of the Creative Commons Attribution License, which permits unrestricted use, distribution, and reproduction in any medium, provided the original author and source are credited.

Source of Support: Nil, Conflict of Interest: None declared. 